\title{
ON KOOPMAN AND PERRON-FROBENIUS OPERATORS OF RANDOM DYNAMICAL SYSTEMS ${ }^{*, * *, * * *}$
}

\author{
MOHAMED HMissi ${ }^{1}$
}

\begin{abstract}
In this paper, we investigate the Koopman and the Perron-Frobenius operators of random dynamical systems in general setting. Moreover, we give some necessary and sufficient conditions for the existence of invariant densities for the associated Perron-Frobenius operators.

Résumé. Dans cet article, nous étudions les opérateurs de Koopman et de Perron-Frobenius associés aux systèmes dynamiques aléatoires en toute généralité. De plus, nous donnons des conditions nécessaires et suffisantes pour l'existence des densités invariantes pour les opérateurs de PerronFrobenius associés.
\end{abstract}

\section{INTRODUCTION}

In the last years, Koopman and Perron-Frobenius operators associated to (deterministic) dynamical systems, are extensively studied (cf. essentially the monographs [4,9]). This is motivated by the interpretation of the asymptotic behavior for such systems, from the statistical point of view. However, for random dynamical systems, this setting is essentially investigated for product of random maps (cf. [3,6] and the references therein). In this paper we investigate the Koopman and the Perron-Frobenius operators for general random dynamical systems. Moreover, we give some characterizations of invariant densities for such operators.

Let $(\Omega, \mathcal{F}, \mathbb{P})$ be a probability space, let $E$ be a Polish space endowed with its $\sigma$-algebra $\mathcal{B}$ and let $\mu$ be a probability measure on $(\Omega \times E, \mathcal{F} \otimes \mathcal{B})$ with marginal $\mathbb{P}$ on $(\Omega, \mathcal{F})$. Then $\mu(d \omega, d x)=\mu_{\omega}(d x) \mathbb{P}(d \omega)$ where $\left(\mu_{\omega}\right)$ is an essentially unique family of probabilities on $(E, \mathcal{B})$. This factorization of $\mu$ will play a fundamental role throughout the present paper.

A random dynamical system (with base space $\Omega$ and taking values in $E$ ) consists of a pair $(\theta, \varphi)$ where $\theta: \mathbb{T} \times \Omega \rightarrow \Omega$ is a translation and $\varphi: \mathbb{T} \times \Omega \times E \rightarrow E$ is a cocycle. The skew product associated to $(\theta, \varphi)$, is the map $\Phi: \mathbb{T} \times \Omega \times E \rightarrow \Omega \times E$ defined by $\Phi(t)(\omega, x):=(\theta(t) \omega, \varphi(t, \omega) x)$. If $\Phi$ is nonsingular with respect to $\mu$ then, the Koopman and the Perron-Frobenius operators $K_{\Phi}: L^{\infty}(\mu) \rightarrow L^{\infty}(\mu)$ and $T_{\Phi}: L^{1}(\mu) \rightarrow L^{1}(\mu)$ are well defined. $K_{\Phi}$ and $T_{\Phi}$ are said to be associated to the random dynamical system $(\theta, \varphi)$.

\footnotetext{
* MSC (2010): Primary: 37A40. Secondary: 47B38; $47 A 35$

** Key words: dynamical system, random dynamical system, factorization, random equation, Koopman operator, PerronFrobenius operator, invariant measure, invariant density.

*** Mots clefs: système dynamique, système dynamique aléatoire, factorisation, équation aléatoire, opérateur de Koopman , opérateur de Perron-Frobenius, mesure invariante, densité invariante.

1 Département de Mathématiques, Faculté des Sciences de Tunis, Université de Tunis Elmanar, TN-2092 Elmanar, Tunis, Tunisia. Med.Hmissi@fst.rnu.tn 
The paper is organized as follows.

In the first section, we give the background required to establish our main results. According to [9], Sections 3 and 7 (cf. also [4]), we introduce the classical notions of Koopman and Perron-Frobenius operators associated to a nonsingular transform and we outline briefly some of their fundamental properties. Then, we recall the notion of dynamical system and we associate the (semi)groups of Koopman and Perron-Frobenius operators. In the second section, we present first the notion of random dynamical system following [1], Sections 1 and 2. Moreover, we give some useful Lemmas which specify preserving (or nonsingular) measures in terms of their factorizations. In the last section, we prove some existence characterizations of $T_{\Phi}$-invariant densities. In fact, we deal with $\mathbb{P}$-preserving $\theta$, with two-sided time $\mathbb{T}$ and with random Dirac or tensor product measure.

For random Dirac measure $\mu$ (i.e. $\left.\mu_{\omega}=\delta_{Y(\omega)}\right)$, we prove that a density $\beta \in L^{1}(\mu)$ is $T_{\Phi}$-invariant if and only if $\omega \mapsto \beta(\omega, Y(\omega))$ is $T_{\theta}$-invariant. If $\mu=\mathbb{P} \otimes \rho$ (for some probability $\rho$ on $(E, \mathcal{B})$ ), then $\beta$ is $T_{\Phi}$-invariant if and only if, for $t \in \mathbb{T}$, for $\rho$-a.e. $x \in E$ and for $\mathbb{P}$-a.e. $\omega \in \Omega$

$$
T_{\theta(-t)} \beta(., x)(\omega)=T_{\varphi(t, \omega)} \beta(\omega, .)(x) .
$$

Finally, we suppose in addition that, $E$ is the unit cube in $\mathbb{R}^{d}$ and $x \mapsto \varphi(t, \omega) x$ is a $\mathcal{C}^{1}$-diffeomorphism. In this case, $\beta$ is $T_{\Phi}$-invariant if and only if

$$
\beta(\omega, x)=\left|J_{\varphi(t, \omega)}(x)\right| \cdot K_{\Phi(t)} \beta(\omega, x)
$$

for all $t \in \mathbb{T}$, for $\rho$-a.e. $x \in E$ and for $\mathbb{P}$-a.e. $\omega \in \Omega$.

The paper contains also some comments, examples and applications of these results.

\section{Preliminaries}

Let $(\Gamma, \mathcal{G}, \mu)$ be a probability space, let $L^{1}(\mu)$ the Banach space of all (class of) $\mu$-integrable real-valued functions defined on $\Gamma$ with the $L^{1}$-norm $\|f\|_{1}=\int|f| d \mu$, and let $L^{\infty}(\mu)$ the space of all bounded (class of) measurable functions on $\Gamma$ with the $L^{\infty}$-norm $\|g\|_{\infty}=$ ess sup $|g|$. Denote by $I_{\Gamma}$ the identity operator of $\Gamma$. A function $f \in L^{1}(\mu)$ is called density if $f \geq 0$ and $\int f d \mu=1$.

Let $\phi: \Gamma \rightarrow \Gamma$ be a measurable transformation.

A set $A \in \mathcal{G}$ is said to be

(1) $\phi$-invariant if $\phi^{-1}(A)=A, \mu$-a.e. (almost everywhere)

(2) $\mu$-trivial if $\mu(A)=0$ or $\mu(A)=1$

Let $\phi \mu$ be the image measure of $\mu$ by $\phi$, i.e.

$$
\phi \mu(A):=\mu\left(\phi^{-1}(A)\right) ; \quad(A \in \mathcal{G}) .
$$

$\phi$ is said to be

(1) invertible if $\phi$ is one-to-one and $\phi^{-1}$ is measurable.

(2) $\mu$-preserving if $\phi \mu=\mu$.

(3) $\mu$-ergodic if $\phi$ is $\mu$-preserving and each $\phi$-invariant set is $\mu$-trivial.

(4) $\mu$-nonsingular if $\phi \mu$ is absolutely continuous with respect to $\mu$ (i.e. $\phi \mu<<\mu$ ).

\subsection{Koopman and Perron-Frobenius operators}

Let $\phi$ be a $\mu$-nonsingular transformation of the probability space $(\Gamma, \mathcal{G}, \mu)$.

Definition 1.1. The Koopman operator $K_{\phi}$ associated to $\phi$ (with respect to $\mu$ ) is defined by

$$
K_{\phi} f:=f \circ \phi ; \quad\left(f \in L^{\infty}(\mu)\right)
$$


In the definition of the Koopman operator, the condition " $\phi \mu$-nonsingular" is essential; otherwise, $K_{\phi}$ is not well-defined. This can be easily seen by the following simple example.

Example 1.2. Let $\Gamma:=[0,1]$ endowed with its Lebesgue measure $\mu$ and take $\phi(x)=1 / 2$ for every $x \in[0,1]$. Further, let $f(x)=g(x)=1$ for all $x \in[0,1] \backslash\{1 / 2\}$ and let $f(1 / 2)=1$ and $g(1 / 2)=0$.

Clearly $f=g$, $\mu$-a.e. but $K_{\phi} f(x)=1$ and $K_{\phi} g(x)=0$ for each $x \in[0,1]$. Thus, the operator $K_{\phi}$ is not well-defined.

Let us return to the general case: The following facts about Koopman operators hold.

(1) $K_{\phi}$ is a linear operator on $L^{\infty}(\Gamma)$.

(2) $K_{\phi}$ is a contraction of $L^{\infty}(\Gamma)$, i.e. $\left\|K_{\phi} f\right\|_{\infty} \leq\|f\|_{\infty}$ for $f \in L^{\infty}(\Gamma)$.

(3) $K_{\phi}$ is multiplicative, i.e. $K_{\phi}(f g)=K_{\phi} f \cdot K_{\phi} g$ for all $f, g \in L^{\infty}(\mu)$.

(4) $\phi$ is $\mu$-preserving if and only if $\mu$ is $K_{\phi}$-invariant (i.e. $\int K_{\phi} f d \mu=\int f d \mu$ for each $f \in L^{\infty}(\Gamma)$ ).

Recall now the definition of Perron-Frobenius operators: For each $f \in L^{1}(\mu)$, define the measure $\nu$ on $(\Gamma, \mathcal{G})$ by

$$
\nu(A):=\int_{\phi^{-1}(A)} f d \mu ; \quad(A \in \mathcal{G}) .
$$

The assumption $\phi \mu<<\mu$ implies $\nu<<\mu$. Hence there exists by the Radon-Nikodym theorem, a unique function, $f_{\phi} \in L^{1}(\mu)$ such that

$$
\nu(A)=\left(f_{\phi} \cdot \mu\right)(A)=\int_{A} f_{\phi} d \mu ; \quad(A \in \mathcal{G}) .
$$

It follows that the mapping $T_{\phi}: L^{1}(\mu) \rightarrow L^{1}(\mu), f \mapsto f_{\phi}$ is well defined.

Definition 1.3. $T_{\phi}$ is called the Frobenius-Perron operator associated to $\phi$ (with respect to $\mu$ ).

$T_{\phi}$ is implicitly defined by the formula

$$
\int_{A} T_{\phi} f d \mu=\int_{\phi^{-1}(A)} f d \mu ; \quad\left(A \in \mathcal{G}, f \in L^{1}(\mu)\right) .
$$

$T_{\phi}$ is also called transfer operator or Ruelle-Frobenius-Perron Operator of $\phi$.

Let us recall some properties of $T_{\phi}$.

(1) Since the characteristic functions are dense in $L^{\infty}(\mu)$, then (1.3) is equivalent to

$$
\int g T_{\phi} f d \mu=\int f K_{\phi} g d \mu ; \quad\left(f \in L^{1}(\mu), g \in L^{\infty}(\mu)\right)
$$

In other words $T_{\phi}$ is the dual of $K_{\phi}$ with respect to $\mu$. Sometimes, we write (1.4) as below

$$
<T_{\phi} f, g>_{\mu}=<f, K_{\phi} g>_{\mu} ; \quad\left(f \in L^{1}(\mu), g \in L^{\infty}(\mu)\right) .
$$

(2) In particular, $\phi$ is $\mu$-preserving if and only if the constant function 1 is $T_{\phi}$-invariant (i.e. $T_{\phi} 1=1$ ).

(3) $T_{\phi}$ is a Markov operator on $L^{1}(\mu)$, that is $T_{\phi}$ is linear, $T_{\phi} f \geq 0$ and $\left\|T_{\phi} f\right\|_{1}=\|f\|_{1}$ whenever $f \in L^{1}(\Gamma)$ and $f \geq 0$.

(4) $T_{\phi}$ is a contraction of $L^{1}(\mu)$.

(5) $T_{\phi}$ is weakly continuous, i.e. if for $f_{n}, f \in L^{1}(\mu)$, we have $<f_{n}, g>_{\mu} \rightarrow<f, g>_{\mu}$ for all $g \in L^{\infty}(\mu)$, then $<T_{\phi} f_{n}, g>_{\mu} \rightarrow<T_{\phi} f, g>_{\mu}$ for all $g \in L^{\infty}(\mu)$.

(6) If $\phi$ is invertible and $\mu$-preserving, then $T_{\phi}$ is the Koopman operator associated to $\phi^{-1}$, i.e.

$$
K_{\phi} g=g o \phi ; \quad T_{\phi} f=f o \phi^{(-1)} .
$$


Remark 1.4. Following [9] (Theorem 4.1.1), a density $f \in L^{1}(\mu)$ is a fixed point of $T_{\phi}\left(\right.$ i.e. $\left.T_{\phi} f=f\right)$ if and only if the measure $\mu_{f}$ defined by $\mu_{f}(A):=\int_{A} f(x) \mu(d x) ;(A \in \mathcal{G})$, is invariant under $\phi$, that is, $\phi \mu_{f}=\mu_{f}$. In others words, absolutely continuous invariant measures correspond to fixed densities for the corresponding Perron-Frobenius operator.

Thus, the study of the spectrum of the Perron-Frobenius operators, is a fundamental problem in the framework of dynamical systems. For the importance of this problem and many applications, see the monographs [4,9].

\subsection{Dynamical systems}

Throughout the paper, time $\mathbb{T}$ always stands for the following (additive) semigroups or groups, namely $\mathbb{Z}, \mathbb{Z}^{+}, \mathbb{R}$ or $\mathbb{R}^{+}$. $\mathbb{T}$ is always endowed with its Borel $\sigma$-algebra $\mathcal{B}(\mathbb{T})$. $\mathbb{T}$ is said to be two-sided if $\mathbb{T}=\mathbb{Z}$ or $\mathbb{R}$. A family $(\phi(t))_{t \in \mathbb{T}}$ of transformations of $(\Gamma, \mathcal{G}, \mu)$ is called a dynamical system (notation DS) with time $\mathbb{T}$, if

(1) $(t, \omega) \mapsto \phi(t) \omega$ is $(\mathcal{B}(\mathbb{T}) \otimes \mathcal{F}, \mathcal{F})$-measurable.

(2) $\phi$ satisfies the translation equation, i.e. for all $\omega \in \Omega ; t, s \in \mathbb{T}$

$$
\phi(0) \omega=\omega ; \quad \phi(s+t) \omega=\phi(s) \phi(t) \omega .
$$

A DS denoted by $\left(\Gamma, \mathcal{G}, \mu,(\phi(t))_{t \in \mathbb{T}}\right.$ ) (or shortly by $(\phi(t))$ or $\left.\phi\right)$ is said to be

3. $\mu$-nonsingular if $\phi(t)$ is $\mu$-nonsingular for each $t \in \mathbb{T}$.

4. $\mu$-preserving if $\phi(t)$ is $\mu$-preserving for each $t \in \mathbb{T}$.

Example 1.5. Let $\Omega:=\left\{\omega: \mathbb{R} \rightarrow \mathbb{R}^{d}\right.$ such that $\left.\omega(0)=0\right\}$ equipped with the compact open topology, let $\mathcal{F}:=\mathcal{B}(\Omega)$ the associated Borel $\sigma$-algebra and for $t \in \mathbb{R}$, let $W_{t}: \Omega \rightarrow \mathbb{R}^{d}, \omega \mapsto \omega(t)$. There exists, by a classical result (cf. [2], section 8 for example), a unique probability measure $\mathbb{P}$ on $(\Omega, \mathcal{F})$ such that the process $\left(W_{t}\right)_{t \in \mathbb{R}}$ is with stationary and independent increments and $\left(W_{t}-W_{s}\right)$ has the normal distribution with mean 0 and variance $|t-s| \cdot I_{\mathbb{R}^{d}}$. For each $t \in \mathbb{R}$ define the Wiener shift $\theta(t): \Omega \rightarrow \Omega$ by

$$
\theta(t) \omega(s):=\omega(s+t)-\omega(t) ; \quad(\omega \in \Omega, s, t \in \mathbb{R})
$$

It can be easily verified that $\theta$ is a $\mathbb{P}$-preserving dynamical system, called Wiener or Brownian DS.

The Wiener DS is the appropriate sample space in order to interpret stochastic differential equations (SDE) as random dynamical systems (RDS) (cf. the next section). However, SDE are closely related to the notion of (Itô) stochastic integral $\int_{0}^{t} \tau(s, \omega) d W_{s}(\omega)$ (cf. the monograph [10]) for example).

Notice that (1.8) implies that the Wiener process $\left(W_{t}\right)$ satisfies (cf. [5])

$$
W_{s+t}(\omega)-W_{t}(\omega)=\left(W_{s} \circ \theta(t)\right)(\omega) \quad(\omega \in \Omega, s, t \in \mathbb{R}) .
$$

The so called helix equation (1.9), is fundamental in order to prove that, solutions of SDE are RDS.

Let $\left(\Gamma, \mathcal{G}, \mu,(\phi(t))_{t \in \mathbb{T}}\right)$ be a nonsingular DS. By the preceding paragraph, we may associate the families $\left(K_{\phi(t)}\right)_{t \in \mathbb{T}}$ and $\left(T_{\phi(t)}\right)_{t \in \mathbb{T}}$ of Koopman operators and Perron-Frobenius operators respectively. More precisely

$$
K_{\phi(t)} f=f \circ \phi(t) ; \quad\left(t \in \mathbb{T}, f \in L^{\infty}(\mu)\right)
$$

and

$$
\int g \cdot T_{\phi(t)} f d \mu=\int f \cdot g \circ \phi(t) d \mu ; \quad\left(t \in \mathbb{T}, f \in L^{1}(\mu), g \in L^{\infty}(\mu)\right) .
$$

Obviously by $(1.10),\left(K_{\phi(t)}\right)_{t \in \mathbb{T}}$ is a (semi) group of contractions on $L^{\infty}(\mu)$, i.e.

$$
K_{\phi(s+t)}=K_{\phi(s)} \circ K_{\phi(t)} ; \quad(s, t \in \mathbb{T}) .
$$


Using (1.12) and (1.11), we establish the same relation for $\left(T_{\phi(t)}\right)_{t \in \mathbb{T}}$, that is

$$
T_{\phi(s+t)}=T_{\phi(s)} \circ T_{\phi(t)} ; \quad(s, t \in \mathbb{T}) .
$$

Thus, $\left(T_{\phi(t)}\right)_{t \in \mathbb{T}}$ is a contraction (semi) group of Markov operators on $L^{1}(\mu)$.

Remark 1.6. If $\mathbb{T}$ is discrete (i.e. $\mathbb{T}=\mathbb{Z}$ or $\mathbb{Z}^{+}$), then, by putting $\psi:=\phi(1)$, we get $\phi(n)=\psi^{n}$ for each $n \in \mathbb{T}$. In this case, the DS is said to be discrete and generated by $\psi$ and it is denoted by $(\Gamma, \mathcal{G}, \mu, \psi)$.

Moreover, the DS $\left(\psi^{n}\right)$ is $\mu$-nonsingular (resp. $\mu$-preserving) if and only if $\psi$ is $\mu$-nonsingular (resp. $\mu$ preserving). Further, (1.12) and (1.13) become

$$
K_{\phi(n)}=K_{\psi}^{n} ; \quad T_{\phi(n)}=T_{\psi}^{n} ; \quad(n \in \mathbb{T}) .
$$

For a discrete DS, the study of the Koopman and the Perron-Frobenius operators, is therefore reduced to the investigation of the same operators of its generator $\psi$.

\section{Applications to RANDOM DYNAMicAl Systems}

Next, we will always work with the product space $\Gamma:=\Omega \times E$ where $(\Omega, \mathcal{F}, \mathbb{P})$ is a probability space and $E$ is a Polish space (i.e. a complete metrizable and separable space) endowed with its $\sigma$-algebra $\mathcal{B}$. The product space $\Omega \times E$ is always endowed with the product $\sigma$-algebra $\mathcal{F} \otimes \mathcal{B}$.

For $\beta: \Omega \times E \rightarrow \mathbb{R},(\omega, x) \mapsto \beta(\omega, x)$, denote by

$$
\beta_{x}:=\beta(., x) ; \quad \beta_{\omega}:=\beta(\omega, .) ; \quad(\omega \in \Omega, x \in E) .
$$

For $f: \Omega \rightarrow \mathbb{R}$ and $u: E \rightarrow \mathbb{R}$, denote by $f \otimes u$ the tensor product of $f$ and $u$, that is

$$
(f \otimes u)(\omega, x):=f(\omega) \cdot u(x) ; \quad(\omega \in \Omega, x \in E) .
$$

\subsection{Disintegration of measures}

The ergodic theory of random dynamical systems deals with probability measures $\mu$ on $(\Omega \times E, \mathcal{F} \otimes \mathcal{B})$ with marginal $\mathbb{P}$ on $(\Omega, \mathcal{F})$, that is

$$
\mu(F \times E)=\mathbb{P}(F) ; \quad(F \in \mathcal{F}) .
$$

In the sequel, denote by $\mathcal{P}_{\mathbb{P}}(\Omega \times E)$ the set of such measures.

Let $\mu \in \mathcal{P}_{\mathbb{P}}(\Omega \times E)$. The disintegration (or factorization) of $\mu$ with respect to $\mathbb{P}$ is given by the following classical result; cf. [1] (Proposition 1.4.3) and the references therein.

Lemma 2.1. There exists a unique $(\mathbb{P}$-a.s) function $\mu .():. \Omega \times \mathcal{B} \rightarrow[0,1]$ such that

(1) $\omega \mapsto \mu_{\omega}(B)$ is $\mathcal{F}$-measurable for each $B \in \mathcal{B}$.

(2) $B \mapsto \mu_{\omega}(B)$ is a probability on $(E, \mathcal{B})$ for $\mathbb{P}$-a.e. $\omega \in \Omega$.

(3) For every $\mu$-integrable function $\xi$

$$
\int_{\Omega \times E} \xi(\omega, x) \mu(d \omega, d x)=\int_{\Omega}\left(\int_{E} \xi(\omega, x) \mu_{\omega}(d x)\right) \mathbb{P}(d \omega) .
$$

The following particular and important cases, will be investigated later.

(1) $\mu$ is random Dirac measure, i.e. for $\mathbb{P}$-a.e. $\omega \in \Omega, \mu_{\omega}=\delta_{Y(\omega)}$ (the Dirac measure at point $Y(\omega)$ ) where $Y: \Omega \rightarrow \mathbb{R}$ is a random variable.

In this case, $Y$ is called the random factor of the random Dirac measure $\mu$. 
(2) $\mu$ is tensor product, i.e. $\mu:=\mathbb{P} \otimes \rho$ where $\rho$ is a probability on $(E, \mathcal{B})$. The factorization of $\mu$ is trivially given by $\mu_{\omega}=\rho$ for each $\omega \in \Omega$.

Remark 2.2. Let $A \in \mathcal{F} \otimes \mathcal{B}$. It follows from (2.1) that

$$
\mu(A)=\int_{\Omega}\left(\int_{E} 1_{A}(\omega, x) \mu_{\omega}(d x)\right) \mathbb{P}(d \omega) .
$$

Now let $A_{\omega}:=\{x \in E:(\omega, x) \in A\}$ be the $\omega$-section of $A$. (2.2) can be written as

$$
\mu(A)=\int_{\Omega} \mu_{\omega}\left(A_{\omega}\right) \mathbb{P}(d \omega) .
$$

For $\mu$-integrable function $\xi:=f \otimes u$, the formula (2.1) becomes

$$
\int_{\Omega \times E} f(\omega) u(x) \mu(d \omega, d x)=\int_{\Omega} f(\omega)\left(\int_{E} u(x) \mu_{\omega}(d x)\right) \mathbb{P}(d \omega) .
$$

\subsection{Random dynamical system}

For the next definitions and examples, we refer the reader to the monograph [1], Sections 1. and 2.

Definition 2.3. Let $(\Omega, \mathcal{F}, \mathbb{P})$ be a probability space and let $(E, \mathcal{B})$ be a Polish space. A measurable random dynamical system (RDS) with state space $E$ is a pair $(\theta, \varphi)$ consisting of two objects:

(1) A dynamical system $\theta$ defined on the probability space $(\Omega, \mathcal{F}, \mathbb{P})$.

(2) A cocycle $\varphi$ on $E$ over $\theta$, i.e. a $(\mathcal{B}(\mathbb{T}) \times \mathcal{F} \times \mathcal{B}, \mathcal{B})$-measurable map

$$
\varphi: \mathbb{T} \times \Omega \times E \rightarrow E,(t, \omega, x) \mapsto \varphi(t, \omega, x)
$$

such that the family $\varphi(t, \omega):=\varphi(t, \omega,):. E \rightarrow E$ of random mappings, satisfies the cocycle equation, i.e. for $s, t \in \mathbb{T}, \omega \in \Omega$

$$
\varphi(0, \omega)=I_{E}, \quad \varphi(s+t, \omega)=\varphi(s, \theta(t) \omega) \circ \varphi(t, \omega) .
$$

Let $(\theta, \varphi)$ be a RDS on $E$. For each $t \in \mathbb{T}$, define $\Phi(t): \Omega \times E \rightarrow \Omega \times E$ by

$$
\Phi(t)(\omega, x):=(\theta(t) \omega, \varphi(t, \omega) x) ; \quad(\omega \in \Omega, x \in E) .
$$

$(\Phi(t))_{t \in \mathbb{T}}$ is the skew product associated to the $\operatorname{RDS}(\theta, \varphi)$.

Remarks 2.4. (1) If $\Omega$ is reduced to one point, then the cocycle equation is reduced to the translation equation. Indeed, if $\Omega:=\{\varpi\}$ then $\theta_{t} \varpi=\varpi$ for $t \in \mathbb{T}$. Therefore, by putting $\varphi(t):=\varphi(t, \varpi)$, the relation (2.5) becomes

$$
\varphi(0)=I_{E}, \quad \varphi(s+t)=\varphi(s) \circ \varphi(t)
$$

which is the translation equation on $E$.

Hence, RDS generalize in a natural way the deterministic DS.

(2) Using (1.7) and (2.5), it can be easily seen that $\Phi$ is a DS on the product space $(\Omega \times E, \mathcal{F} \otimes \mathcal{B})$. Therefore, the skew product $\Phi$ allows natural generalizations from the deterministic case to the random case.

(3) If $\mathbb{T}$ is two-sided, then by the cocycle equation (2.5), all random mappings $\varphi(t, \omega)$ are invertible and

$$
\varphi(t, \omega)^{-1}=\varphi(-t, \theta(t) \omega) ; \quad(t \in \mathbb{T}, \omega \in \Omega) .
$$


Examples 2.5. (1) Discrete case: Suppose that $\mathbb{T}$ is discrete (i.e. $\mathbb{T}=\mathbb{Z}$ or $\mathbb{Z}^{+}$) and put $\psi:=\phi(1)$ and $\alpha(\omega):=\varphi(1, \omega)$ for $\omega \in \Omega$. In this case, the associated cocycle $\varphi$ is explicitly given by

$$
\varphi(n, \omega)=\alpha\left(\psi^{n-1} \omega\right) \circ \cdots \circ \alpha(\omega) ; \quad(\omega \in \Omega, n \geq 1) .
$$

If $\mathbb{T}=\mathbb{Z}$, we have in addition

$$
\varphi(n, \omega)=\alpha\left(\psi^{n} \omega\right)^{-1} \circ \cdots \circ \alpha\left(\psi^{-1} \omega\right)^{-1} ; \quad(\omega \in \Omega, n \leq-1) .
$$

Conversely, if $\psi$ and $\alpha$ are given, we may associate a unique cocycle $\varphi$ solution of the random difference equation:

$$
x_{n+1}(\omega)=\alpha\left(\psi^{n} \omega\right) x_{n}(\omega) ; \quad x_{0}=x \in E ; n \in \mathbb{T} .
$$

(2) Absolutely continuous case: Let $(\theta(t))_{t \in \mathbb{R}}$ be a DS defined on $(\Omega, \mathcal{F}, \mathbb{P})$, let $E$ be an open set of $\mathbb{R}^{d}$ and let $h: \Omega \times E \rightarrow E$ be measurable and such that for each $\omega \in \Omega,(t, x) \rightarrow h(\theta(t) \omega, x)$ is continuous and $x \rightarrow h(\theta(t) \omega, x)$ is locally-lipschitz for each $t \in \mathbb{R}$. Then the random differential equation

$$
d X_{t}(\omega)=h\left(\theta(t) \omega, X_{t}\right) d t ; \quad X_{0}(\omega)=x \in E
$$

admits a unique solution $\varphi$ which is a $\operatorname{RDS}$ over $\theta$. The solution is given implicitly by

$$
\varphi(t, \omega) x=x+\int_{0}^{t} h(\theta(s) \omega, \varphi(s, \omega) x) d s ; \quad(t \in \mathbb{R}, \omega \in \Omega, x \in E) .
$$

In particular, $t \mapsto \varphi(t, \omega) x$ is absolutely continuous.

(3) Stochastic case: Let $(\Omega, \mathcal{F}, \mathbb{P}, \theta)$ be the Wiener DS on $\mathbb{R}^{d}$. Consider the stochastic differential equation (notation SDE)

$$
d X_{t}=b\left(X_{t}\right) d t+\sigma\left(X_{t}\right) d W_{t} ; \quad X_{0}=x \in \mathbb{R}^{d}
$$

where $b: \mathbb{R}^{d} \rightarrow \mathbb{R}^{d}$ and $\sigma: \mathbb{R}^{d} \rightarrow \mathbb{R}^{d} \times \mathbb{R}^{d}$ are such that

$$
|b(x)-b(y)|+|\sigma(x)-\sigma(y)| \leq D|x-y| ; \quad x, y \in \mathbb{R}^{d}
$$

for some constant $D>0$. Then (2.13) admits a unique solution $\varphi: \mathbb{R} \times \Omega \times \mathbb{R}^{d} \rightarrow \mathbb{R}^{d}$ which is a continuous RDS on $\mathbb{R}^{d}$, over the Wiener DS $\theta$.

Before we define and investigate the Koopman and Perron-Frobenius operators for RDS, we need first to present the following useful results.

Let $(\theta, \varphi)$ be a RDS and let $\Phi$ be the associated skew product. Let $\mu \in \mathcal{P}_{\mathbb{P}}(\Omega \times E)$ and let $(\omega, A) \mapsto \mu_{\omega}(A)$ be the factorization of $\mu$ with respect to $\mathbb{P}$. We check Equation (2.2) on product sets $F \otimes B$ where $F \in \mathcal{F}, B \in \mathcal{B}$. We obtain

$$
\mu(F \times B)=\int_{F} \mu_{\omega}(B) \mathbb{P}(d \omega) .
$$

On the other hand, let $A:=\Phi(t)^{-1}(F \times B)$ and for $\omega \in \Omega$, let $A_{\omega}:=\{x \in E:(\omega, x) \in A\}$ be the $\omega$-section of $A$. From (2.6), it can be easily seen that

$$
A_{\omega}=\left\{\begin{array}{cc}
\varphi(t, \omega)^{-1}(B) & \text { if } \omega \in \theta(t)^{-1}(F) \\
\emptyset & \text { otherwise. }
\end{array}\right.
$$

Hence, by (1.1) and (2.3), we have

$$
\begin{aligned}
\Phi(t) \mu(F \times B) & =\mu\left(\Phi(t)^{-1}(F B)\right) \\
& =\int_{\theta(t)^{-1}(F)} \mu_{\omega}\left(\varphi(t, \omega)^{-1}(B)\right) \mathbb{P}(d \omega) .
\end{aligned}
$$


Using (1.1) again, we get

$$
\Phi(t) \mu(F \times B)=\int_{\theta(t)^{-1}(F)}\left(\varphi(t, \omega) \mu_{\omega}\right)(B) \mathbb{P}(d \omega) .
$$

We deduce first the following result.

Lemma 2.6. If $\theta$ is $\mathbb{P}$-nonsingular and if $\varphi(\omega,$.$) is \mu_{\omega}$-nonsingular for $\mathbb{P}$-a.e. $\omega \in \Omega$, then $\Phi$ is $\mu$-nonsingular.

Proof. Suppose that $\mu(F \times B)=0$, then (2.15) implies that, either $\mathbb{P}(F)=0$ or $\mu_{\omega}(B)=0$ a.e. If $\mathbb{P}(F)=0$ then $\mathbb{P}\left(\theta(t)^{-1}(F)\right)=0$ since $\theta(t)$ is $\mathbb{P}$-nonsingular and thus $\Phi(t) \mu(F \times B)=0$ by $(2.16)$. If $\mu_{\omega}(B)=0$ a.e., then $\left(\varphi(t, \omega) \mu_{\omega}(B)\right)=0$ for $\mathbb{P}$-a.e. $\omega \in \Omega$, by the hypothesis and $(2.16)$ implies again that $\Phi(t) \mu(F \times B)=0$.

Further, if $\theta$ is $\mathbb{P}$-preserving and if $\mathbb{T}$ is two-sided, then (2.15) reads

$$
\mu(F \times B)=\int_{\theta(-t)(F)} \mu_{\theta(t) \omega}(B) \mathbb{P}(d \omega)
$$

and (2.16) becomes

$$
\Phi \mu(F \times B)=\int_{\theta(-t)(F)}\left(\varphi(t, \omega) \mu_{\omega}\right)(B) \mathbb{P}(d \omega) .
$$

As a consequence of (2.17) and (2.18), we immediately have the following Lemma whose proof can be also found in [1], Theorem 1.4.5.

Lemma 2.7. If $\theta$ is $\mathbb{P}$-preserving and if $\mathbb{T}$ is two-sided time, then $\Phi$ is $\mu$-preserving if and only if

$$
\varphi(t, \omega) \mu_{\omega}=\mu_{\theta(t) \omega} ; \quad(t \in \mathbb{T}, \text { a.e } \omega \in \Omega) .
$$

\subsection{Koopman and Perron-Frobenius operators for RDS}

Let $(\theta, \varphi)$ be a $\operatorname{RDS}$ on $(\Omega, \mathcal{F}, \mathbb{P})$ with state space $(E, \mathcal{B})$ and let $\Phi$ be the associated skew product. Let $\mu \in \mathcal{P}_{\mathbb{P}}(\Omega \times E)$ and let $(\omega, A) \mapsto \mu_{\omega}(A)$ the factorization of $\mu$ with respect to $\mathbb{P}$.

The following definitions are adapted from [3].

Definitions 2.8. Suppose that the skew product $\Phi$ is $\mu$-nonsingular.

(1) The Koopman operator of $(\theta, \varphi)$ (with respect to $\mu$ ) is by definition the Koopman operator $K_{\Phi}$ induced by the associated skew product $\Phi$, i.e. (cf. (1.2))

$$
K_{\Phi(t)} \xi(\omega, x)=\xi \circ \Phi(t)(\omega, x)=\xi(\theta(t) \omega, \varphi(t, \omega) x)
$$

for $t \in \mathbb{T}, \omega \in \Omega, x \in E$ and $\left.\xi \in L^{\infty}(\mu)\right)$.

(2) The Perron-Frobenius operator of $(\theta, \varphi)$ is by definition the Perron-Frobenius operator $T_{\Phi}$ induced by $\Phi$, i.e. $T_{\Phi(t)}: L^{1}(\mu) \rightarrow L^{1}(\mu)$ is the unique Markov operator defined implicitly, for $\beta \in L^{1}(\mu), \xi \in L^{\infty}(\mu)$ by

$$
\int_{\Omega \times E} \xi T_{\Phi(t)} \beta d \mu=\int_{\Omega \times E} \beta K_{\Phi(t)} \xi d \mu
$$

where $K_{\Phi}$ is the Koopman operator of the $\operatorname{RDS}(\theta, \varphi)$.

In view of Lemma 2.6, it is worthwhile to note that, the Koopman and the Perron-Frobenius operators of the $\operatorname{RDS}(\theta, \varphi)$ are well defined (with respect to $\mu$ ) whenever the analogous operators are well defined for all transforms $\theta(t)$ with respect to $\mathbb{P}$ and a.e. for the transforms $\varphi(t, \omega)$ with respect to the factor probability $\mu_{\omega}$.

Using the factorization formula (2.1), the implicit expression (2.21) becomes

$$
\int_{\Omega}\left(\int_{E} \xi(\omega, x) T_{\Phi(t)} \beta(\omega, x) \mu_{\omega}(d x)\right) \mathbb{P}(d \omega)=\int_{\Omega}\left(\int_{E} \beta(\omega, x) K_{\Phi(t)} \xi(\omega, x) \mu_{\omega}(d x)\right) \mathbb{P}(d \omega) .
$$


Taking $\xi:=f \otimes u \in L^{\infty}(\mu)$ and using (2.20), we get

$$
\int_{\Omega} f(\omega)\left(\int_{E} u(x)\left(T_{\Phi(t)} \beta\right)(\omega, x) \mu_{\omega}(d x)\right) \mathbb{P}(d \omega)=\int_{\Omega} f(\theta(t) \omega)\left(\int_{E} u(\varphi(t, \omega) x) \beta(\omega, x) \mu_{\omega}(d x)\right) \mathbb{P}(d \omega) .
$$

In what follows, the last formula (2.22), will be used in order to characterize the $T_{\Phi}$-invariant densities.

Recall first that $\Phi$ is $\mu$-preserving if and only if the constant function 1 is a $T_{\Phi}$-invariant density. This follows immediately from the duality formula (2.21).

\section{EXISTENCE OF $T_{\Phi}$-INVARIANT DENSITIES}

In this last section, we suppose that $\mathbb{T}=\mathbb{Z}$ or $\mathbb{R}$ and $\theta$ is $\mathbb{P}$-preserving (i.e. $\theta(t) \mathbb{P}=\mathbb{P}$ for each $t \in \mathbb{T}$ ). In the last paragraph, we will comment briefly these two additional hypotheses. Moreover, we will consider random Dirac measures and tensor product measures.

In this case, each $\theta(t)$ is invertible and $\theta(t)^{-1}=\theta(-t)$. Hence, by (1.6) we get

$$
T_{\theta(t)} f=f \circ \theta(-t) ; \quad\left(t \in \mathbb{T}, f \in L^{1}(\mathbb{P})\right) .
$$

In particular, a density $f \in L^{1}(\mathbb{P})$ is $T_{\theta(-t)}$-invariant if and only if $f$ is $T_{\theta(t)}$-invariant.

\subsection{Random Dirac measure case}

Suppose that $\mu$ is random Dirac measure and let $Y$ be the random factor of $\mu$ (i.e. $\mu_{\omega}=\delta_{Y(\omega)}$ for $\mathbb{P}$-a.e. $\omega \in \Omega$ ).

Next, denote by $\left.\left(I_{\Omega}, Y\right): \Omega \rightarrow \Omega \times E\right), \omega \mapsto(\omega, Y(\omega))$. Note first that, for $q=1$ or $\infty$

$$
\xi \in L^{q}(\mu) \Leftrightarrow \xi \circ\left(I_{\Omega}, Y\right) \in L^{q}(\mathbb{P})
$$

and for $\xi \in L^{1}(\mu), \beta \in L^{\infty}(\mu)$

$$
<\xi, \beta>_{\mu}=<\xi \circ\left(I_{\Omega}, Y\right), \beta \circ\left(I_{\Omega}, Y\right)>_{\mathbb{P}} .
$$

Let $(\theta, \varphi)$ be a RDS and let $\Phi$ be the associated skew product. In this case, $(2.22)$ becomes

$$
\int f(\omega) u(Y(\omega))\left(T_{\Phi(t)} \beta\right)(\omega, Y(\omega)) \mathbb{P}(d \omega)=\int f(\theta(t) \omega) u(\varphi(t, \omega) Y(\omega)) \beta(\omega, Y(\omega)) \mathbb{P}(d \omega)
$$

which is valuable if $f, u \circ Y \in L^{\infty}(\mathbb{P}), \beta \in L^{1}(\mu)$ and $t \in \mathbb{T}$.

The following technical result is an immediate consequence of Lemma 2.7 (cf. [1], Remark 1.4.6).

Lemma 3.1. $\Phi$ is $\mu$-preserving if and only if $Y$ is random fixed point of $(\theta, \varphi)$, i.e.

$$
\varphi(t, \omega) Y(\omega)=Y(\theta(t) \omega) ; \quad(t \in \mathbb{T}, \text { a.e } \omega \in \Omega) .
$$

Now we present the first result about the existence of $T_{\Phi}$-invariant densities.

Theorem 3.2. Suppose that $\Phi$ is $\mu$-preserving, then a density $\beta \in L^{1}(\mu)$ is $T_{\Phi}$-invariant if and only if

$$
\beta(\omega, Y(\omega)=\beta(\theta(t) \omega, Y(\theta(t) \omega)) ; \quad(t \in \mathbb{T}, \text { a.e } \omega \in \Omega)
$$

i.e., $\omega \mapsto \beta(\omega, Y(\omega))$ is $T_{\theta}$-invariant.

Moreover, if in addition $\theta$ is $\mathbb{P}$-ergodic, then the constant 1 is the unique $T_{\Phi}$-invariant density. 
ProOF. From (3.3), it is straightforward that a density $\beta \in L^{1}(\mu)$ is $T_{\Phi}$-invariant if and only if

$$
\int f(\omega) u(Y(\omega)) \beta(\omega, Y(\omega)) \mathbb{P}(d \omega)=\int f(\theta(t) \omega) u(\varphi(t, \omega) Y(\omega)) \beta(\omega, Y(\omega)) \mathbb{P}(d \omega)
$$

for all $f, u \circ Y \in L^{\infty}(\mathbb{P})$ and $t \in \mathbb{T}$.

On the other hand, since $\theta$ is $\mathbb{P}$-preserving then

$$
\int f(\omega) u(Y(\omega)) \beta(\omega, Y(\omega)) \mathbb{P}(d \omega)=\int f(\theta(t) \omega) u(Y(\theta(t) \omega)) \beta(\theta(t) \omega, Y(\theta(t) \omega)) \mathbb{P}(d \omega)
$$

Combining (3.6) and (3.7), we deduce that $\beta$ is $T_{\Phi}$-invariant if and only if

$$
u(\varphi(\omega) Y(\omega)) \beta(\omega, Y(\omega))=u(Y(\theta(t) \omega)) \beta(\theta(t) \omega, Y(\theta(t) \omega))
$$

for all $u$ such that $u \circ Y \in L^{\infty}(\mathbb{P})$, for all $t \in \mathbb{T}$ and for $\mathbb{P}$-a.e. $\omega \in \Omega$.

Using Lemma3.1 (relation (3.4)), (3.8) and (3.1), we conclude that $\beta \circ\left(I_{\Omega}, Y\right)$ is $T_{\theta}$-invariant.

If in addition $\theta$ is $\mathbb{P}$-ergodic then $\beta \circ\left(I_{\Omega}, Y\right)$ is necessary constant and therefore equal to 1 since $\int \beta \circ\left(I_{\Omega}, Y\right) d \mathbb{P}=1$.

Examples 3.3. $\quad(1)$ Let $(\Omega, \mathcal{F}, \mathbb{P}, \psi)$ be an ergodic discrete DS. Let $a \in]-1,1\left[, b \in L^{1}(\mathbb{P})\right.$ and let $\alpha$ : $\Omega \times \mathbb{R} \rightarrow \mathbb{R}$ defined by $\alpha(\omega, x)=a x+b(\omega)$. Define

$$
Y(\omega)=\sum_{n=0}^{n=\infty} a^{n} \cdot b\left(\psi^{(-n-1)} \omega\right), \quad(\omega \in \Omega)
$$

It can be easily verified that $Y$ is the unique random fixed point of the $\operatorname{RDS}(\psi, \alpha)$. We deduce from Theorem 3.2 that 1 is the unique invariant density for the associated Perron-Frobenius operator with respect to the random Dirac measure with random factor $Y$.

(2) Let $(\Omega, \mathcal{F}, \mathbb{P}, \theta)$ be an ergodic DS with time $\mathbb{T}=\mathbb{R}$ and let $A: \Omega \rightarrow \mathbb{R}^{2 d}, b: \Omega \rightarrow \mathbb{R}^{d}$ be two $\mathbb{P}$-integrable random variables. Under some convenient conditions (cf. [1], Section 5), the RDS generated by the random differential equation

$$
d X_{t}=\left(A(\theta(t) \omega) X_{t}(\omega)+b(\theta(t) \omega)\right) d t ; \quad X_{0}=x \in \mathbb{R}^{d}
$$

admits a unique random fixed point given by

$$
Y(\omega)=\int_{-\infty}^{0} \vartheta(-t, \theta(t) \omega) b(\theta(t)) d t
$$

where $\vartheta$ is the cocycle defined by the linear random differential equation

$$
d X_{t}=\left(A(\theta(t) \omega) X_{t}(\omega)\right) d t ; \quad X_{0}=x \in \mathbb{R}^{d}
$$

By Theorem 3.2, 1 is the unique $T_{\Phi}$-invariant density with respect to the random Dirac measure defined by $Y$.

(3) The unique solution of the affine one-dimensional SDE

$$
d X_{t}=\left(1-\frac{1}{2} X_{t}\right) d t+X_{t} d W_{t} ; \quad X_{0}=x \in \mathbb{R}
$$


is given by

$$
\varphi(t, \omega) x=x \exp \left(-t+W_{t}(\omega)\right)+\int_{0}^{t} \exp \left(s-t+W_{t}(\omega)-W_{s}(\omega)\right) d s
$$

which is a $\operatorname{RDS}$ over the Wiener $\operatorname{DS}(\Omega, \mathcal{F}, \mathbb{P}, \theta)$. (In fact, using only (1.9), it can be directly verified that the mapping $\varphi$ defined by (3.11) is a cocycle over $\theta$. In particular, no knowledge from SDE theory, are needed for this example).

Now we replace $\omega$ by $\theta(-t) \omega)$ in (3.11) and we tend $t \rightarrow \infty$. Using (1.9) again, the obtained limit

$$
Y(\omega):=\int_{-\infty}^{0} \exp \left(s-W_{s}(\omega)\right) d s
$$

is the unique random fixed point for the $\operatorname{RDS}(\theta, \varphi)$. We conclude once again that 1 is the unique $T_{\Phi}$-invariant density with respect to the associated random Dirac measure.

(4) Following ( [11], Theorem 2.2), there exists a large class of RDS which possesses a unique random fixed point. Hence, for such RDS, the constant 1 the unique $T_{\Phi}$-invariant density for the associated the random Dirac measure.

(5) Let $\varphi: \mathbb{R} \times \Omega \times[-1,1] \rightarrow[-1,1]$ be the cocycle, over the Wiener DS $\theta$, given by

$$
\varphi(t, \omega) x=x \exp \left(t+W_{t}(\omega)\right) \cdot\left(1-x^{2}+x^{2} \exp \left(2 t+2 W_{t}(\omega)\right)\right)^{1 / 2} .
$$

In fact, $\varphi$ is the solution of the SDE (cf. [8])

$$
d X_{t}=\frac{3}{2} X_{t}\left(X_{t}-X_{t}^{2}\right)^{2} d t+\left(X_{t}-X_{t}^{3}\right) d W_{t} ; \quad X_{0}=x \in[-1,1]
$$

From (3.14), it can be easily verified that the random fixed points of $(\theta, \varphi)$ are all discrete random variables $Y$ with support in $\{-1,0,1\}$. So, let $a, b, c \geq 0, a+b+c=1, Y$ with law $\mathcal{L}(Y)=a \delta_{-1}+b \delta_{0}+c \delta_{1}$ and $\mu$ the associated random Dirac measure. Using Theorem 3.2, it follows that a density $\beta \in L^{1}(\mu)$ is $T_{\Phi}$-invariant if and only if $a \beta_{-1}+b \beta_{0}+c \beta_{1}$ is $T_{\theta}$-invariant.

\subsection{Tensor product case}

In this paragraph, we suppose that $\mu$ is a tensor product, i.e. $\mu:=\mathbb{P} \otimes \rho$ where $\rho$ is a probability on $(E, \mathcal{B})$. The factorization of $\mu$ is trivially given by $\mu_{\omega}=\rho$ for each $\omega \in \Omega$. This situation was already studied for some particular RDS (cf. [6] and the related references).

Let $(\theta, \varphi)$ be a $\operatorname{RDS}$ and let $\Phi$ be the associated skew product.

Lemma 3.4. $\Phi$ is $\mathbb{P} \otimes \rho$-nonsingular if and only if $\theta$ is $\mathbb{P}$-nonsinglar and $\varphi(., \omega)$ is $\rho$-nonsingular for $\mathbb{P}$-a.e. $\omega \in \Omega$.

Proof. In view of Lemma 2.7, it is enough to prove the sufficient condition. Let $F \in \mathcal{F}$ and $B \in \mathcal{B}$. In this case (2.15) becomes

$$
\mu(F \times B)=\int_{F} \rho(B) \mathbb{P}(d \omega)
$$

and (2.16) is reduced to

$$
\Phi \mu(F \times B)=\int_{\theta^{-1}(F)}(\varphi(\omega, .) \rho)(B) \mathbb{P}(d \omega) .
$$

Suppose first that $\rho(B)=0$. From $(3.15)$ we deduce that $\mu(\Omega \times B)=0$ and then $\Phi(\mathbb{P} \otimes \rho)(\Omega \times B)=0$ since $\Phi(\mathbb{P} \otimes \rho)<<\mathbb{P} \otimes \rho$. Therefore, we get $(\varphi(\omega,.) \rho(B))=0$ for $\mathbb{P}$-a.e. $\omega \in \Omega$ by $(3.16)$. 
Suppose now that $\mathbb{P}(F)=0$. By the same argument as before, we get $(\mathbb{P} \otimes \rho)(F \times E)=0$. Hence $\Phi(\mathbb{P} \otimes \rho)(F \times E)=0$ and then $\mathbb{P}\left(\theta^{-1}(F)\right)=0$ by $(3.16)$.

Now we come to the second result about the existence of $T_{\Phi}$-invariant densities.

Theorem 3.5. Suppose that $\Phi$ is $\mathbb{P} \otimes \rho$-preserving. Then a density $\beta \in L^{1}(\mathbb{P} \otimes \rho)$ is $T_{\Phi}$-invariant if and only if

$$
T_{\theta(-t)} \beta_{x}(\omega)=T_{\varphi(t, \omega)} \beta_{\omega}(x) ; \quad(t \in \mathbb{T}, \text { a.e } \omega \in \Omega, x \in E) .
$$

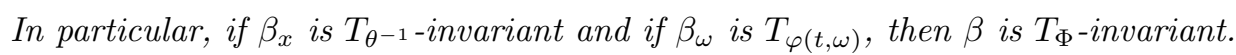

Proof. Note first that, since $\Phi$ is $\mathbb{P} \otimes \rho$-preserving (hence $\mathbb{P} \otimes \rho$-nonsingular), Lemma 3.4 implies that $\varphi(t, \omega)$ are $\rho$-nonsingular.

Let $\beta \in L^{1}(\mathbb{P} \otimes \rho)$ be a density. By $(2.22), \beta$ is $T_{\Phi}$-invariant if and only if

$$
\int f(\omega)\left(\int u(x) \beta(\omega, x) \rho(d x)\right) \mathbb{P}(d \omega)=\int f(\theta(t) \omega)\left(\int u(\varphi(t, \omega) x) \beta(\omega, x) \rho(d x)\right) \mathbb{P}(d \omega)
$$

for all $t \in \mathbb{T}, f \in L^{\infty}(\mathbb{P})$ and $u \in L^{\infty}(\rho)$.

On the other hand, since $\theta$ is $\mathbb{P}$-preserving then

$$
\int f(\omega)\left(\int u(x) \beta(\omega, x) \rho(d x)\right) \mathbb{P}(d \omega)=\int f(\theta(t) \omega)\left(\int u(x) \beta(\theta(t) \omega, x) \rho(d x)\right) \mathbb{P}(d \omega) .
$$

Combining again (3.18) and (3.19) and repeating the same arguments as in the proof of Theorem 3.2, we see that $\beta$ is $T_{\Phi}$-invariant if and only if

$$
\int u(x) \beta(\theta(t) \omega, x) \rho(d x)=\int u(\varphi(t, \omega) x) \beta(\omega, x) \rho(d x)
$$

for all $t \in \mathbb{T}, u \in L^{\infty}(\rho)$ and $\mathbb{P}$-a.e. $\omega \in \Omega$. However, by the definition of the Koopman operator, it is obvious that (3.20) is equivalent to

$$
\int u(x) \beta(\theta(t) \omega, x) \rho(d x)=\int K_{\varphi(t, \omega)} u(x) \beta(\omega, x) \rho(d x) .
$$

Now, since $\mathbb{T}$ is two-sided and $\theta$ is $\mathbb{P}$-preserving then, using (3.1), (3.21) reads

$$
\int u(x) T_{\theta(-t)} \beta_{x}(\omega) \rho(d x)=\int K_{\varphi(t, \omega)} u(x) \beta(\omega, x) \rho(d x) .
$$

Since $u$ is arbitrary, the result follows from (3.22) using the duality relation (1.4) applied to $K_{\varphi(t, \omega)}$ and the associated Perron-Frobenius operator $T_{\varphi(t, \omega)}$.

Examples to which this theorem can be applied are provided by the following corollary.

Corollary 3.6. Let $f \in L^{1}(\mathbb{P})$ and $u \in L^{1}(\rho)$ be two densities. Then $f \otimes u$ is a T $T_{\Phi}$-invariant if and only if $f$ is $T_{\theta}$-invariant and $u$ is $T_{\varphi(., \omega)}$-invariant for $\mathbb{P}$-a.e. $\omega \in \Omega$.

Proof. Let $\beta:=f \otimes u$, then $\beta_{\omega}=f(\omega) \cdot u$ and $\beta_{x}=u(x) \cdot f$. Hence by linearity of $T_{\theta}$ and $T_{\varphi(t, \omega)}$ we get

$$
T_{\theta(-t)}(f \otimes u)_{x}(\omega)=u(x) \cdot T_{\theta(-t)} f(\omega)
$$

and

$$
T_{\varphi(t, \omega)}(f \otimes u)_{\omega}(x)=f(\omega) \cdot T_{\varphi(t, \omega)} u(x) .
$$


On the other hand, the $T_{\Phi}$-invariance of $f \otimes u$ reads

$$
T_{\Phi}(f \otimes u)(\omega, x)=f(\omega) \cdot u(x) .
$$

The desired result is obtained by combining (3.17), (3.23), (3.24) and (3.25).

In what follows and to keep things manageable, we focus on the specific case where the phase space $E$ is the unit cube in $\mathbb{R}^{d}$ with Lebesgue measure $\rho(d x):=d x$. This setting has been studied extensively for particular RDS (cf. [6] and the references therein). Moreover, we suppose that $x \mapsto \varphi(t, \omega)$ is a $\mathcal{C}^{1}$-diffeomorphism for all $\omega \in \Omega$ and $t \in \mathbb{T}$ and denote by $J_{\varphi(t, \omega)}$ its Jacobian.

Let us first give the following Lemma.

Lemma 3.7. Let $\beta \in L^{1}(\mathbb{P} \otimes \rho)$ be a density and let $\nu(d \omega, d x):=\beta(\omega, x) \mathbb{P}(d \omega) \rho(d x)$.

Then $\nu \in \mathcal{P}_{\mathbb{P}}(\Omega \times E)$ if and only if $\int_{E} \beta(\omega, x) \rho(d x)=1$ for $\mathbb{P}$-a.e. $\omega \in \Omega$.

Moreover, in this case $\nu_{\omega}=\beta_{\omega} \cdot \rho$ for $\mathbb{P}$-a.e. $\omega \in \Omega$.

Proof. For $F \in \mathcal{F}$ and $B \in \mathcal{B}$, we get

$$
\left.\nu(F \times B)=\int_{F} \int_{B} \beta(\omega, x) \rho(d x)\right) \mathbb{P}(d \omega) .
$$

Taking $B=E$ in (3.26), we obtain immediately the first part of the Lemma.

Comparing (3.26) and (2.15), we deduce that $\nu_{\omega}=\beta_{\omega} \cdot \rho$ for $\mathbb{P}$-a.e. $\omega \in \Omega$.

We conclude this paper by the following characterization which allows immediate applications if the cocycle $\varphi$ is explicitly given.

Theorem 3.8. Suppose that $\varphi(., \omega)$ is $\rho$-nonsingular for $\mathbb{P}$-a.e. $\omega \in \Omega$.

Let $\beta \in L^{1}(\mathbb{P} \otimes \rho)$ be a density such that $\int_{E} \beta(\omega, x) \rho(d x)=1$ for $\mathbb{P}$-a.e. $\omega \in \Omega$. Then $\beta$ is $T_{\Phi}$-invariant if and only if

for all $t \in \mathbb{T}$, for $\rho$-a.e. $x \in E$ and for $\mathbb{P}$-a.e. $\omega \in \Omega$.

$$
\beta(\omega, x)=\left|J_{\varphi(t, \omega)}(x)\right| \cdot K_{\Phi(t)} \beta(\omega, x)
$$

In particular, the constant 1 is $T_{\Phi}$-invariant if and only if $\left|J_{\varphi(t, \omega)}\right|=1$ for all $t \in \mathbb{T}$ and for $\mathbb{P}$-a.e $\omega \in \Omega$.

Proof. Define $\nu(d \omega, d x):=\beta(\omega, x) \mathbb{P}(d \omega) \rho(d x)$ the measure of density $\beta$ with respect to $\mathbb{P} \otimes \rho$. By Lemma 3.7, $\nu \in \mathcal{P}_{\mathbb{P}}(\Omega \times E)$ and $\nu_{\omega}=\beta_{\omega} \cdot \rho$ for $\mathbb{P}$-a.e. $\omega \in \Omega$. Therefore, for each open subset $B$ of $E$ and for all $t \in \mathbb{T}$ and $\mathbb{P}$-a.e. $\omega \in \Omega$, yields

$$
\nu_{\theta(t) \omega)}(B)=\int_{B} \beta(\theta(t) \omega, x) d x .
$$

On the other hand, by using (2.7), we get

$$
\begin{aligned}
\left(\varphi(t, \omega) \nu_{\omega}\right)(B) & =\nu_{\theta(t) \omega)}\left(\varphi(t, \omega)^{-1}(B)\right) \\
& =\int_{\varphi(-t, \theta(t) \omega)(B)} \beta(\omega, x) d x .
\end{aligned}
$$

Hence, by standard change of variables

$$
\left(\varphi(t, \omega) \nu_{\omega}\right)(B)=\int_{B} \beta(\omega, \varphi(-t, \theta(t) \omega)(x)) \cdot\left|J_{\varphi(-t, \theta(t) \omega)}(x)\right| d x
$$

Now, $\beta$ is $T_{\Phi}$-invariant if and only if $\Phi$ is $\nu$-preserving. According to Lemma 2.7 , this is equivalent to

$$
\varphi(t, \omega) \nu_{\omega}=\nu_{\theta(t) \omega} ; \quad(t \in \mathbb{T}, \text { a.e. } \omega \in \Omega)
$$


From (3.28), (3.29) and (3.30), we get

$$
\beta(\theta(t) \omega, x)=\left|J_{\varphi(-t, \theta(t) \omega)}(x)\right| \cdot \beta(\omega, \varphi(-t, \theta(t) \omega) x
$$

for all $t \in \mathbb{T}$, for $\rho$-a.e. $x \in E$ and for $\mathbb{P}$-a.e. $\omega \in \Omega$. The result follows immediately if we replace $\theta(t) \omega$ by $\omega$ and $(-t)$ by $t$ in the relation (3.31).

\subsection{Final remarks}

In this paper, we have made some hypotheses in order to characterize $T_{\Phi}$-invariant densities. We have essentially supposed that $\mathbb{T}$ is two-sided, $\theta$ is $\mathbb{P}$-preserving and $\mu$ is a random Dirac measure or a tensor product measure.

(1) If $\theta$ is $\mathbb{P}$-preserving (and $\mathbb{T}$ is not necessary two-sided), then $\Phi$ is $\mu$-preserving for some $\mu \in \mathcal{P}_{\mathbb{P}}(\Omega \times E)$ if and only if (cf. [1], Theorem 1.4.5).

$$
\mathbb{E}\left(\varphi(t, .) \mu . \mid \theta(t)^{-1}(\mathcal{F})\right)=\mu_{\theta(t) .} ; \quad(\mathbb{P}-\text { a.e. })
$$

where $\mathbb{E}$ is the expectation operator associated to $\mathbb{P}$.

Using (3.32), it seems possible (and important) to generalize the tree characterizations proved in this paper.

It is also worthwhile to obtain similar results if $\mu$ is neither Dirac nor tensor product.

(2) If $\theta$ is (only) $\mathbb{P}$-nonsingular, relations of type (3.4) or (3.32) are not known to the knowledge of the author. However, characterizations of $T_{\Phi}$-invariant densities, is still an important project in this case.

\section{REFERENCES}

[1] Arnold L.: Random Dynamical Systems, Springer-Verlag, Berlin (1998)

[2] Billingsley P.: Convergence of Probability Measures, Wiley Series in probability and Statistics, Chicago, Second Edition (1999)

[3] Bogenschuetz T., Kowalski Z.S.: Exacteness of skew products with expanding fibre maps, Studia Mathematica 120 (2), 159-168 (1996)

[4] Ding J., Zhou A.: Statical properties of deterministic systems, Springer-Verlag (2009)

[5] Hmissi M., ben Salah I., Taouil H.: On the Helix Equation, in: Fournier-Prunaret D. et al.(eds.), European Conference on Iteration Theory, Nant 2010, Esaim: Proceedings, Vol. 36, 197-208 (2012)

[6] Hsieh Shelley L.Y.: Ergodic Theory of Multidimensional Random Dynamical Systems, Thesis, University of Victoria (2008)

[7] Karatzs I., Shreve S.T.: Brownian Motion and Stochastic Calculus, Springer, New York, Second Edition (1991)

[8] Kloeden P.E., Platen E.: Numerical Solution of Stochastic Differential Equations, Springer, Berlin (1992)

[9] Lasota A., Mackey M.C.: Chaos, Fractals, and Noise: Stochastic Aspects of Dynamics, Second Edition, Springer-Verlag (1994)

[10] Oksendal B.: Sochastic Differential Equations, Sixth Edition, Springer-Verlag (2005)

[11] Schmalfuss B.: A Random Fixed Point Theorem Based on Lyapunov Exponents, Random and Computational Dynamics 4 (4), 257-268 (1996) 